\title{
Integration of a new position sensor based on four quadrant fiber optic bundle to measure the cantilever deflections in atomic force microscopy head
}

\author{
Younes Boukellal $^{1, a}$ and Sebastien Ducourtieux ${ }^{1}$ \\ ${ }^{1}$ French National Metrology Institute / Laboratoire National de Métrologie et d'Essais (LNE)-29 avenue Roger Hennequin Trappes/ France
}

\begin{abstract}
Résumé. Cet article présente l'implémentation d'une tête pour microscope à Force Atomique intégrant un capteur original en remplacement à une photodiode quatre quadrants dans la méthode du levier optique. Ce capteur est composé d'un bundle de fibres à quatre quadrants permettant de mesurer la déflexion du levier tout en s'affranchissant des sources de chaleurs liées à l'électronique de conditionnement de la méthode de mesure. Le bundle est constitué de 40000 microfibres avec un arrangement customisé permettant d'obtenir une surface à quatre quadrants identiques en entrée. Avant le développement du premier prototype, le bundle de fibres a été modélisé et évalué expérimentalement par comparaison à une photodiode quatre quadrants [1]. A partir des données de modélisation et des données expérimentales, la réponse des deux capteurs est similaire. Comme le capteur a été développé pour son utilisation en AFM, il a été intégré dans une tête AFM et a été testé en conditions réelles d'utilisations dans le but de générer les premières images AFM. Des courbes d'approches en mode tapping et contact ont également été obtenues afin d'évaluer la linéarité et la sensibilité du bundle de fibres aux déflexions du levier.
\end{abstract}

Fiber optic, Fiber optic bundle, atomic force microscopy, optical beam deflection method, displacement sensor, tapping mode, contact mode.

\section{INTRODUCTION}

Atomic force microscope (AFM) was introduced by Binnig, Quate, and Gerber as a method for high resolution topographic imaging of both insulators and conductors. In AFM, the sample to be imaged is brought close to a sensing tip attached to a small cantilever. Interaction forces between sample and tip deflect the cantilever. Cantilever deflections can be measured using various techniques: optical beam deflection, interferometry selfsensing methods. For quantitative imaging, it is necessary to understand the system used for detection of cantilever deflection and torsion. In most AFMs the sensing system for cantilever motion is used as a null detector. Consequently the quality of the measurement and its accuracy depends critically on the detection system. The most widespread method is the optical beam deflection method where light is reflected from the cantilever onto the centre of a quadrant photodiode (QPD). The operation of such a position sensing detector is quite simple. A laser beam with Gaussian spot falls on the centre of the detector. By combining the signal detected by $\mathrm{A}, \mathrm{B}, \mathrm{C}$ and $\mathrm{D}$ quadrants, the displacement of the laser spot relative to the centre of the quadrant detector can be approximated by using the following formula :

$$
\text { Equation 1: }\left\{\begin{array}{l}
x=\frac{(B+D)-(A+C)}{\operatorname{sum}}=\frac{(B+D)-(A+C)}{A+B+C+D} \\
y=\frac{(A+B)-(C+D)}{\operatorname{sum}}=\frac{(A+B)-(C+D)}{A+B+C+D}
\end{array}\right.
$$

Also, the related intensity variation is normalized by the sum intensity of the four quadrants given by $\mathrm{A}+\mathrm{B}+\mathrm{C}+\mathrm{D}$. to take into account any possible fluctuation of the laser beam intensity. However for low noise applications, it is necessary to integrate the photodiode very close from its conditioning electronic circuit or even directly on the circuit. This implies several heat sources induced by the conditioning circuit itself which could disturb the deflection measurement or even the overall behaviour of the instrument (thermal dilatation, thermal drift...).This is specifically the case in the field of dimensional nanometrology in which instruments are supposed to work at $20^{\circ} \mathrm{C}$ and where any heat sources can critically affect the thermal and dimensional stability of the instrument by creating local thermal gradient. To counter this, the QPD and its conditioning electronic circuit placed inside the instrument has been replaced by a thermically passive sensor. The aim of our approach was to externalize the heating system from the instrument itself by using optical fibers and by mimicking the functioning principle of the QPD used for position sensing applications. After several investigations, this led to the development of a first prototype based on quadrant structured fiber optic bundle shown on figure 1 .

\footnotetext{
a Author corresponding email: younes.boukellal@,1ne.fr
} 


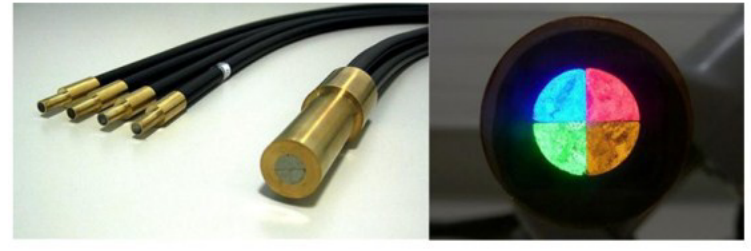

Figure 1. On the left picture, the fiber optic bundle with one common input structured in four quadrants and four outputs. The ferrule serves as a holder. On the right picture, the light of four power LEDs of different colours is injected from the outputs solely to distinguish the quadrants geometry on the input.

The optic fiber bundle has an active surface area of 10 $\mathrm{mm}$ diameter that comprises 40.000 borosilicate's fibers of $50 \mu \mathrm{m}$ core diameter spread over the four quadrants. The gap between quadrants is fixed to $50 \mu \mathrm{m}$ and is similar to quad cell photodiode. Deflection and torsion signals are then processed using a homemade electronic conditioning circuit. Before the integration of the bundle on the AFM, it has been modelled and experimentally evaluated. Results showed that the fiber optic bundle response is very similar to the one of quad cell photodiode. This results have been subject to an article [1].

\section{Integration and test of the bundle on an AFM head}

In order to test the fiber bundle in real conditions, we integrated it temporarily in an AFM developed at LNE. It has a very simple architecture in which it was relatively easy to replace the four-quadrant photodiode and its electronic conditioning circuits by the optic fiber bundle and its conditioning circuit. We also replaced the laser diode by a fibered superluminescente diode (SLD). In this way, the main heat sources in the AFM head has been outsourced.

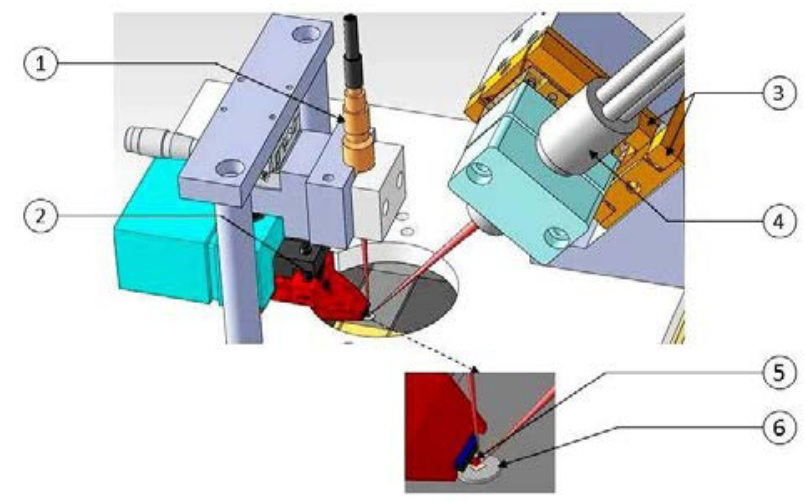

Figure 4: modified AFM head using optical lever method (OBD) to detect the deflection of cantilever. The OBD is based on the use of fiber optic bundle (4) and fibered superluminescente diode (1). The focused laser spot delivered by a fibered superluminescente diode is reflected on the backside of cantilever (5) and the reflected beam is directed onto the surface of quadrant fiber bundle (4) to sense the power variation. A piezo actuator (2) is used for cantilever modulation (tapping mode). Two manual translation stages (3) are used to set the position of the laser spot on the bundle surface.
This setup was used to test the ability of the bundle to correctly detect deflections of the cantilever either in contact mode or tapping mode. For this test, a tip from NCHR NanoWorld has been used. The cantilever is rectangular, measuring 125 micrometers long (lc) and has a stiffness of $42 \mathrm{~N} / \mathrm{m}$. the reflected laser beam length $1_{\mathrm{cp}}$ is $80 \mathrm{~mm}$. With these parameters, it is possible to trace back the movement of the laser spot on the bundle using the following equation

Equation 2:

$\Delta a$ is the laser spot displacement on the bundle surface. Considering a deflection $\Delta z$ about $50 \mathrm{~nm}$, the laser spot displacement $\Delta a$ on the bundle is evaluated to $96 \mu \mathrm{m}$. For a similar displacement, it has been shown in [1] that the bundle response is linear. The first test was performed in order to validate the approach curves either in tapping mode or in contact mode on Silica wafer. This allowed the evaluation of the sensitivity of the deflection measurement system based on the whole SLD + tip/cantilever + optic fiber bundle + electronic conditioning circuit.

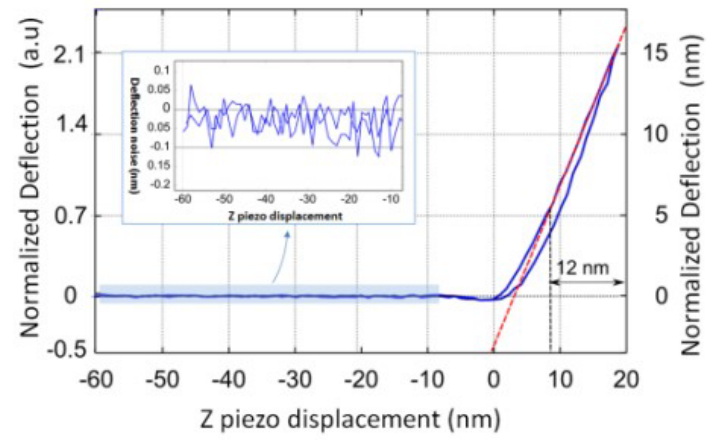

Figure 5. Contact mode approach curve. The conversion of the normalized deflection from the arbitrary unit to $\mathrm{nm}$ unit is done considering the linear slope in the repulsive regime equal to 1 and the substrate hard enough and does not deform.

By analysing the figure 4, one can see that the measurement system can detect a small deflection below $1 \mathrm{~nm}$. The noise level of the deflection measurement on the approach curve before contact (region between -60 and $-10 \mathrm{~nm}$ ) reaches $0.1 \mathrm{~nm}$. From this data and using Equation 2, the noise level of the laser spot fluctuation on the surface of the bundle has been estimated to $192 \mathrm{~nm}$. Using the Hooke's law ( $\mathrm{f}=\mathrm{kz}$ ) and stiffness of the cantilever $\mathrm{k}(42 \mathrm{~N} / \mathrm{m})$, we reach a noise level for measuring force $\mathrm{f}$ of $4.2 \mathrm{nN}$. We reproduced this test in Tapping mode by oscillating the tip at near its resonant frequency $(310 \mathrm{kHz})$. The tapping curve curve approach is shown in the following figure.

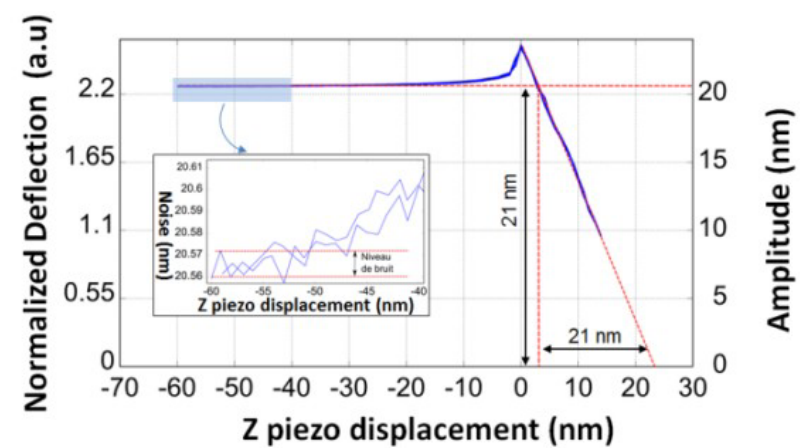


Figure 6. Tapping mode approach curveFor tapping mode, the oscillation amplitude of the tip has not been fully damped with the sample surface to avoid damaging it. The system response has been calibrated in the same way as for the contact mode. The tip oscillation achieved 21 manometers and the noise level is less than $0.01 \mathrm{~nm}$, which is ten times less than in contact mode. This noise reduction results from the use of lock-in amplifier to demodulate the signal.

Finally, a topography images has been performed on two different samples and using Tapping mode. This allowed the validation of the integrated deflection measurement system.
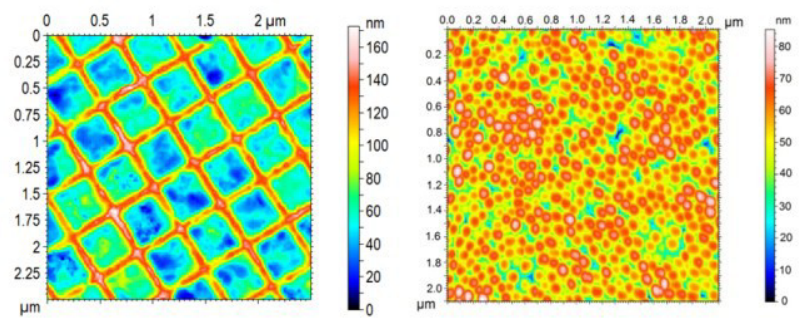

Figure 7. On the left, a $2.5 \mu \mathrm{m} \times 2.5 \mu \mathrm{m}$ image of $2 \mathrm{~d}$ calibration specimen composed of cellulose acetate with periodicity of 2160 lines $/ \mathrm{mm}, 0.463$ micrometers pitch and $31 \mathrm{~nm}$ of step height .On the right, topography image of $\mathrm{SiO} 2$ nanoparticles with an average diameter of $50 \mathrm{~nm}$. The images have been processed using Mountains Map software. These images show that the deflection measurement system based on the use of fiber optic bundle instead of four quadrant photodiodes works perfectly and is capable of delivering a very good quality image

[1]Y. Boukellal and S. Ducourtieux H 2015 Meas. Sci. Technol. 26. 1 doi:10.1088/0957-0233/26/1/015201 